\title{
MDM4 is a rational target for treating breast cancers with mutant p53
}

Panimaya Jeffreena Miranda ${ }^{1,2}$, Daniel Buckley ${ }^{1,2}$, Dinesh Raghu ${ }^{1,2}$, Jia-Min B. Pang ${ }^{1,3}$, Elena A. Takano $^{1,3}$, Reshma Vijayakumaran ${ }^{1,2}$, Amina F.A.S. Teunisse ${ }^{4}$, Atara Posner ${ }^{1,2}$, Tahlia Procter ${ }^{1,2}$, Marco J. Herold ${ }^{5,6}$, Cristina Gamel1 ${ }^{1,2}$, Jean-Christophe Marine ${ }^{7}$, Stephen B. Fox ${ }^{1,3}$, Aart Jochemsen ${ }^{4}$, Sue Haupt ${ }^{1,2^{* \#}}$ and Ygal Haupt ${ }^{1,2,8,9 *}$

${ }^{1}$ Tumour Suppression Laboratory, Peter MacCallum Cancer Centre, Melbourne, Victoria 3000, Australia

${ }^{2}$ The Sir Peter MacCallum Department of Oncology, The University of Melbourne, Victoria 3010, Australia

${ }^{3}$ Department of Pathology, Peter MacCallum Cancer Centre, Melbourne, 3000, Victoria, Australia.

${ }^{4}$ Department of Molecular Cell Biology, University Medical Centre, Leiden, The Netherlands

${ }^{5}$ Molecular Genetics of Cancer, The Walter and Eliza Hall Institute, Parkville, Victoria, Australia

${ }^{6}$ Department of Medical Biology, University of Melbourne, Parkville, Victoria 3050, Australia

${ }^{7}$ Center for Human Genetics, K U Leuven, Leuven, Belgium

${ }^{8}$ Department of Pathology, The University of Melbourne, Parkville 3010 Victoria, Australia

${ }^{9}$ Department of Biochemistry and Molecular Biology, Monash University, Clayton, 3168, Australia

\# Correspondence to: Sue Haupt, Tumour Suppression Laboratory, Peter MacCallum Cancer Centre, 305 Grattan Street, Melbourne, Victoria, Australia 3000.E-mail: Sue.Haupt@petermac.org

* Contributed equally as senior authors

\section{Running Title: MDM4 is a rational target for treating breast cancers with mutant p53}

The authors declare no conflict of interest.

This is the author manuscript accepted for publication and has undergone full peer review but has not been through the copyediting, typesetting, pagination and proofreading process, which may lead to differences between this version and the Version of Record. Please cite this article as doi: $10.1002 /$ path.4877

This article is protected by copyright. All rights reserved. 


\section{ABSTRACT}

Mutation of the key tumour suppressor p53 defines a transition in the progression toward aggressive and metastatic breast cancer (BC) with the poorest outcome. Specifically, p53 mutation frequency exceeds $50 \%$ in Triple Negative BC (TNBC). Key regulators of mutant p53 that facilitate its oncogenic functions are potential therapeutic targets. We report here that the MDM4 protein is frequently abundant in the context of mutant p53 in basal-like BC samples. Importantly, we show that MDM4 plays a critical role in the proliferation of these BC cells. We demonstrate that conditional knockdown (KD) of MDM4 provokes growth inhibition across a range of breast cancer subtypes with mutant p53, including, luminal, Her2+ and TNBCs. In vivo, MDM4 was shown to be crucial for the establishment and progression of tumours. This growth inhibition was mediated at least in part by the cell cycle inhibitor p27. Depletion of p27 together with MDM4 KD led to a recovery of the proliferative capacity of cells that were growth inhibited by MDM4 KD alone. Consistently, we identified low levels of p27 expression in basal-like tumours corresponding to high levels of MDM4 and p53. This predicts a signature for a sub-set of tumours that may be amenable to targeted therapies toward MDM4 and mutant p53. The therapeutic potential of MDM4 as a target in BC with mutant p53 was shown in vitro using a small molecule inhibitor. Overall, our study supports MDM4 as a novel therapeutic target for BC expressing mutant $\mathrm{p} 53$.

Keywords: MDM4, breast cancer, mutant p53, p27, TNBC

This article is protected by copyright. All rights reserved. 


\section{INTRODUCTION}

Breast cancer $(\mathrm{BC})$ is the most commonly diagnosed cancer in women worldwide and a major cause of female cancer morbidity [1]. BC is a heterogeneous disease that is divided into subtypes according to molecular signatures [2]. Relapse of drug resistant, metastatic disease poses the greatest survival risk for BC patients [3]. Aggressive and metastatic BC is driven by mutation of the tumour suppressor p53 [4]. Wild-type (wt) p53 is a pivotal orchestrator of cellular stress responses and a vital tumour suppressor [5]. Wt p53 preserves genomic integrity and prevents cancer by promoting DNA repair or eliminating damaged cells, through elicitation of growth inhibition by cell cycle arrest, apoptosis and senescence [6,7]. Failure of p53 to function properly is a frequent outcome of its mutation, leading to cancer development. Mutation in p53 occurs at an overall incidence of $~ 23 \%$ in all $\mathrm{BC}$ [8], but is more prevalent in Triple Negative BC (TNBC; negative for oestrogen and progesterone receptors and without amplified Her2+). The p53 mutation incidence has been reported as $>50 \%$ in TNBCs [9] and in $84 \%$ of basal-like BCs, of which $80 \%$ were TNBCs [2]. Consistently, TNBCs have a particularly poor prognosis [10]. Effective new treatments are essential to combat aggressive BCs, including TNBCs. Identifying oncogenes that partner with mutant p53 to promote malignant BC is a rational approach to unmask candidate drug targets.

MDM4 (MDMX; HMD4/X) is a close family member of MDM2 and both perform distinct but cooperative functions to negatively regulate wt p53 (reviewed in [11]). MDM4 suppresses wt p53 transcriptional activity. MDM4 lacks E3 ligase activity, but cooperates with the E3 ligase activity of MDM2 to promote p53 ubiquitination and consequent proteasomal degradation. Deregulation of the normal functions of the MDM proteins can perturb wt p53 tumour suppressor function with consequences for cancer onset. When deregulated, MDM4 and MDM2 
function as oncoproteins largely by suppressing wt p53, but also through p53-independent pathways [11].

We recently showed that downregulation of MDM4 using a knockdown (KD) approach in BC, unleashes wt p53 to suppress tumour growth [12]. However, the majority of human cancers, including TNBC/basal-like breast cancer, eventually select for p53 mutations. This raises a key question whether MDM4 has a functional role in BCs with mutant p53, which is the essence of this study. We show that MDM4 protein levels are frequently high in BCs expressing mutant p53. Using a conditional KD system, MDM4 KD inhibits the growth of mutant p53 expressing BC cell lines in vitro and in vivo. Our findings also demonstrate a link between high MDM4/low p27/high mutant p53, which predicts a signature for further stratification of aggressive BCs, with opportunities for therapeutic intervention. Furthermore, we have tested the therapeutic potential of targeting MDM4 in mutant p53 expressing BC using a small molecular inhibitor. 


\section{MATERIALS AND METHODS}

\section{Cell culture and MDM4 inhibitor}

Cell lines (Table S1) as previously described [13] or purchased from ATCC [www.atcc.org] were maintained at $5 \% \mathrm{CO}_{2}$ in Dulbecco's Modified Eagle's Medium (DMEM) with 0.1\% Penicillin/Streptomycin and supplemented with $10 \%$ fetal calf serum (FCS; Gibco - Invitrogen, VIC, Australia). MDM4 inhibitor XI-011 (NSC 146109 hydrochloride; C17H16N2S.HCl; APExBIO Houston, TX) was prepared as previously described [14].

\section{Inducible lentiviral shRNA sequences and viral production.}

The lentiviral vector FH1t with GFP tag [15] was used to generate the short hairpin RNAs as previously [12] (Table S2). Viruses were generated and cells transduced essentially as described [15], with $\mathrm{CaPO}_{4}$ transfection replaced by PEI [12]). shRNA expression in vitro was induced with 10ng/ml Doxycycline (Doxy), with replenishment every two days.

\section{Immunoblotting and flow cytometry}

Immunoblotting was undertaken as previously described [16] with antibodies to human p53 (DO-1; PAb1801; a kind gift of D. Lane); MDM4 (mouse clone 8C6; Millipore, MA, USA; or rabbit polyclonal A300-287; Bethyl laboratories, Montgomery, TX, USA); MDM2 (rabbit; C18; Santa Cruz Biotechnology, USA); HSP60 (rabbit; sc-13966; Santa Cruz Biotechnology); p27 (mouse; Anti-p27, clone 57, BD Biosciences, San Jose, CA, USA ); p21 (rabbit; 12D1, \#2947 CST; Denver, USA); and E cadherin (mouse clone 36, BD Biosciences). For flow cytometry, live cultured cells were harvested at designated times and distinguished from dead cells by exclusion of the nucleic acid stain To-pro-3-iodide (Life Technologies, Thermo Scientific, Australia) using FACSVERSE (BD Biosciences) for cell counting. Cell cycle 
analysis was performed following the addition of $0.1 \%$ Triton $\mathrm{X}-100$ to permeabilise plasma membranes.

\section{Orthotopic tumour studies.}

Female NOD SCID Gamma IL2R-gamma chain (NSG) mice at 6-8 weeks of age were injected with TNBC cells into contralateral mammary fat pads (numbers 4 and 9). MDM4 KD was induced by priming with intraperitoneal (IP) Doxy injection $(40 \mu \mathrm{g} / 100 \mu \mathrm{l})$ and maintained subsequently with Doxy supplementation in the drinking water $(2 \mathrm{mg} / \mathrm{ml})$. Two models were studied: one for tumour establishment, with Doxy administration coincident with tumour cell injection and a second for tumour treatment, with Doxy administration when tumours reached $200 \mathrm{~mm}^{3}$. Tumour growth was measured (Volume $=$ Length $\mathrm{x}$ Width $\mathrm{x}$ Width/2) until the ethical endpoint $\left(\sim 1500 \mathrm{~mm}^{3}\right)$, at which point the mice were killed as per institute guidelines. The research was approved by the Institute's Ethics Committee. Necropsies were performed and tumour and control tissues were harvested and divided for snap freezing in liquid nitrogen for protein analysis or fresh frozen tissues pieces were embedded in OCT for cryotome sectioning and immunofluorescence staining, while other pieces were fixed in $10 \%$ neutral buffered formalin (NBF) prior to immunohistochemistry (IHC).

\section{Microscopy analyses.}

Fixed tissues were processed for paraffin embedding and sectioned. IHC was performed with antibodies to human p53 (mouse monoclonal DO-7; Leica Biosystems, Newcastle-Upon-Tyne, UK, Novocastra); MDM4 (A300 287A; Bethyl Laboratories, TX, USA), MDM2 (rabbit polyclonal C-18; Santa Cruz) and p27 (mouse; clone 57, BD Biosciences). Histopathological analyses were performed by JMP and SF. Images were photographed using a BX-51 microscope with Leica DFC290HD camera and analysed with Leica Application Suite V3.8.0 
software. IHC scoring was as described previously [12]. Staining intensity was evaluated on a scale ranging from 0 to 3 and the proportion of cells stained was evaluated as $0=$ no detectable stain; $1:<10 \%$ stained, $2: 10-50 \%, 3: 50-80 \%$ stained; $4:>80 \%$. A combined histoscore was calculated by adding the value of the intensity to the proportion of cells stained with a final maximum score of 8 .

\section{Colony assay and colorimetric cell enumeration.}

In colony formation, 700 CAMA-1 cells transduced either for shMDM4 or shCtrl were seeded in 6-well plates and cultured in $10 \mathrm{ng} / \mathrm{ml}$ Doxy. After eight days, cells were fixed with 70\% icecold ethanol at $4^{\circ} \mathrm{C}$ for $24 \mathrm{~h}$ and then stained with SRB for $1 \mathrm{~h}$ at room temperature, gently rinsed with distilled water and dried at room temperature [17]. Plates were imaged using an Olympus DP21 camera on an Olympus SZ61 microscope. For cell enumeration, Sulforhodamine B (SRB) assays were performed [18] using a VERSAmax tunable microplate reader (Molecular devices, Orleans Drive, Sunnyvale, CA, USA).

\section{RNA extraction and PCRs.}

RNA was extracted and cDNA generated as previously [12]. Reverse transcriptase (Promega) and semiquatitative reverse transcription (RT)-PCR was performed for MDM4-FL and MDM4$S$ as previously [13]. Real Time PCR analysis of the cDNA was performed as previously [12] using appropriate forward and reverse primers (Hu p27 Fwd 5'GGCCTCAGAAGGACGTGAAAC-3'; Hu p27 Rev 5'-ACAGGATGTCCATTCCATGA-3'; Hu RPL37a Fwd 5'-GCCAGCACGCCAAGTACAC-3'; Hu RPL37a Rev 5'CCCCACAGCTCGTCTCTTCA-3'). The cycle threshold $\left(\mathrm{C}^{\mathrm{T}}\right)$ values were used to calculate the expression of each gene and was normalised with the relative transcript abundance using RPL3a by " CT $=\mathrm{C}^{\mathrm{T}}$ (gene of interest) $-\mathrm{C}^{\mathrm{T}}($ RPL37a). 


\section{RESULTS}

MDM4 protein levels were high in BC biopsies with elevated p53 levels.

High levels of MDM4 protein were found to be a feature of wt p53 BCs, as demonstrated in luminal subtypes. Basal-like biopsies in our mixed BC subtype TMA also stained intensely for p53, generally indicative of p53 mutations, coincident with high levels of MDM4 [12]. To expand this finding, a TMA of 87 biopsies derived from basal-like BC (where the majority have a TNBC phenotype [19]) was stained by IHC for p53 and MDM4 (Figure 1). Similar tumour regions were stained for p53 and MDM4 (with either the mouse monoclonal antibody 8C6 or the rabbit polyclonal MDM4 antibody A300-287, which has been extensively authenticated for IHC by us $[12,20]$ and others (e.g. [21]).

This analysis revealed that $64 \%$ of samples in the basal-like TMA express high levels of p53 compared to adjacent tissue and hence are assumed to represent mutant p53 (Figure 1). MDM4 levels were high in all these samples. This contrasts with the very low staining of MDM2, the other MDM family member and major regulator of wt p53 [22,23]. These results suggest that MDM4 is highly expressed in TNBC/basal-like BCs expressing mutant p53.

\section{MDM4 protein and corresponding $M D M 4-F L$ are present in BCs with mutant p53.}

To further explore the high levels of MDM4 in BC, we chose a set of BC cell lines derived from a range of subtypes (luminal, Her2+ and TNBC) that have p53 mutations defined by sequencing (Table S1). Two wt p53 lines, MCF 10A non-transformed breast epithelial cells and MCF7 luminal BC cells were included for comparison. Levels of the MDM4 protein have been linked with its alternate mRNA splicing $[13,24]$. A $\sim 76 \mathrm{kDa}$ isoform has been designated as full-length (FL): MDM4-FL derived from MDM4-FL mRNA and a short (S) isoform, $M D M 4$ - 
$S$, generated by alternative splicing involving exon 6 excision. The resultant isoform is less stable and poorly detected, if at all, at the protein level [25]. In our panel of mutant p53 BC cell lines, MDM4 was detected as a $\sim 76 \mathrm{kDa}$ isoform, with higher levels in all TNBC lines compared to Her2+ and luminal subtypes (Figure 2A; Figure S1). Immunofluorescence of the TNBC lines MDA-MB-468 and MDA-MB-231 revealed similar patterns for MDM4 and p53 (Figure S2) as observed for the TMAs (Figure 1A). In BC with mutant p53, reverse transcription PCR identified mRNA of not only the short isoform, but also the full-length stable isoform, which contributed to the detected protein (Figure 2B). These experiments demonstrate expression of $M D M 4-F L$ mRNA and its corresponding protein in BCs with mutant $\mathrm{p} 53$, across multiple BC subtypes.

\section{MDM4 KD attenuates the growth of mutant p53 BC cells in vitro}

To measure the effect of MDM4 KD on BC cells expressing mutant p53, we employed lentiviral mediated doxycycline (Doxy)-inducible shRNA directed to MDM4. Initially, the efficiency of four different shRNAs directed to MDM4 and two different shRNA controls (Table S2) were compared by monitoring MDM4 protein using immunoblotting in the luminal BC line with mutant p53, CAMA-1 (Figure S3A). Three of the four shRNAs to MDM4 reduced its levels efficiently; shMDM4\#2 was the least efficient. Subsequent studies were undertaken using shMDM4. The control shRNA sequence used in ongoing studies (shRNA directed to mouse $M d m 4$ sequence) is referred to as shCtrl (Table 2). We then applied MDM4 KD to TNBC lines expressing mutant p53 and studied the biological outcome, with KD confirmed by immunoblotting. For this purpose we examined the effect of MDM4 KD over 7 days on proliferation (measuring live cell dye exclusion by flow cytometry) and MDM4 levels in MDA-MB-468 (Figure 3A,B), MDA-MB-231 (Figure 3C,D) and SUM-159PT (Figure 3E,F). At day 7, growth inhibition in response to MDM4 KD was significant for MDA-MB- 
$468(\mathrm{p}=0.0001)$; MDA-MB-231 ( $\mathrm{p}=0.0003) ; \mathrm{SUM}-159 \mathrm{PT}(\mathrm{p}=0.0002)$ and CAMA-1

$(\mathrm{p}=0.0034)$. In all three TNBC cell lines, MDM4 KD significantly reduced cell numbers, supporting the notion that MDM4 is required for the proliferation of these TNBC cell lines expressing mutant p53. Similarly, we studied the impact of MDM4 KD on the proliferation of luminal line CAMA-1 using flow cytometry (Figure 3D, H) and in a colony assay (9 days; Figure $\mathrm{S} 3 \mathrm{~B}, \mathrm{C}$ ). In addition, the impact of MDM4 KD on cell proliferation was measured using the SRB assay to enumerate cell numbers in the TNBC lines MDA-MB-468, MDA-MB-231 and OCUM-B and in the Her2+ line BT474. These results show that MDM4 KD inhibits the growth of BCs with mutant p53 across luminal, Her2+ and TNBC subtypes (Figure S4).

\section{The growth of mutant p53 BC xenografts is inhibited by MDM4 KD.}

To evaluate whether MDM4 KD impedes BC cell growth in vivo, corresponding to its impact on cultured cells in vitro, we adopted an orthotopic xenograft transplantation approach in female NSG mice. The impact of MDM4 KD was tested across two stages of tumour development of mutant p53 expressing BC cell line-derived xenografts: firstly on tumour establishment and secondly on the progression of already established tumour xenografts. For these studies, TNBC cells expressing either the conditional shMDM4 or shCtrl lentiviral vectors were orthotopically injected into contralateral mammary fat pads (numbers 4 and 9 ) of NSG females ( $n=6$ per treatment). For the establishment studies, mice were administered with Doxy (initially with IP priming injection and subsequently with drinking water supplementation). In control mice (shCtrl), TNBC MDA-MB-468 tumours rapidly established and reached the ethical limits of $1500 \mathrm{~mm}^{3}$ by $\sim 50$ days post injection, at which point they were terminated (Figure 4A,B). MDM4 KD mice only reached ethical end point by $\sim 125$ days, lengthening the survival time by 2.5 fold relative to the control mice (Figure 4B). These findings suggest that in this orthotopic xenograft TNBC model with mutant p53, MDM4 
functions as an oncogenic promoter of tumourigenesis. Furthermore, these studies demonstrate that targetting MDM4 is a rational approach to significantly extend survival.

To mimic treatment of growing tumours, we examined the effect of MDM4 KD on xenografts of MDA-MB-468 (Figure 4C,D) and MDA-MB-231 TNBC cells (Figure 4E,F). Orthotopic xenografts were established for each cell line as described above, with the exception that commencement of Doxy treatment was delayed until tumours reached $200 \mathrm{~mm}^{3}$. Doxy treatment did not impede growth of control tumours, which grew to the ethical end point size by $\sim 50$ days post injection. In contrast, Doxy initially slowed tumour development, followed by growth retardation compared to control mice. This retardation in growth extended the survival of MDM4 KD mice by approximately 2 fold (Figure 4D,F). These findings demonstrate that down-regulation of MDM4 efficiently impedes the growth of an established tumour of transplanted TNBC cells. These studies provide the first evidence for the efficacy of targeting MDM4 in established TNBC tumours expressing mutant $\mathrm{p} 53$.

Despite extended tumour growth retardation and extended survival, in each instance, tumours eventually resurged and reached ethical limits. To study this phenomenon further, MDA-MB-468 xenografted tumours from the treatment model were analysed. At end point the tumours were divided for either formalin fixation and paraffin embedding (FFPE) or for fresh freezing in OCT mounting media. FFPE samples were subjected to haematoxylin and eosin (H\&E) staining to study morphology and IHC for Ki67 (indicative of proliferation), MDM4, p53, MDM2 and p27 (Figure $4 G)$. Fresh frozen samples were cryostat sectioned and examined for green fluorescence that is indicative of the presence of the GFP-marker of shRNA. At the end point, GFP detection was nearly completely absent from the shMDM4 samples, although abundant in the shCtrl. For all other markers, including MDM4, levels were comparable between end point tumours from shMDM4 and shCtrl tumours. These results indicate that selection against shMDM4 is robust in these transformed tumour cells and vital for tumour regrowth. The intolerance of MDM4 KD in these regrown 
tumours and the resurgence of MDM4 detection indicates that MDM4 is a potent oncogenic driver of these BCs with p53 mutation.

\section{Small molecule MDM4 inhibitor impedes the growth of TNBC cells with mutant p53}

The growth inhibitory response of MDM4 KD in BC cells with mutant p53 prompted the expectation that depleting MDM4 chemically may have therapeutic application. The small molecule XI-011 has been reported to target MDM4 [14] by inhibiting its expression. To examine the efficacy of this small molecule inhibitor, the TNBC cells most extensively tested throughout this study (MDA-MB-468 and MDA-MB-231) were treated with XI-011 (0.2 $\mu \mathrm{M})$ over 7 days (with drug replenishment on day 4) and the cellular response was compared to vehicle control (DMSO; Figure 5). The proliferation of both cell lines was significantly inhibited by XI-011 as enumerated by flow cytometry (volumetric counting; $p=0.0018$ for MDA-MB-468 and $p=0.0007$ for MDA-MB-231, Figure 5A,B). Reduced cell numbers corresponded with the depletion of MDM4 expression (Figure 5C, D). These results demonstrate the efficacy of targeting mutant p53 TNBC cells with a small molecule inhibitor of MDM4, at least under in vitro cultured conditions.

\section{P27 is a key target of MDM4 oncogenic activity in BC with mutant p53.}

Identifying targets that MDM4 exploits to promote proliferation in malignant $\mathrm{BCs}$ is crucial to delineate the mechanism of MDM4 oncogenic activities that are independent of wt p53, and also for defining new candidates for therapeutic activation. The cell cycle inhibitors p21 and p27 have been linked to MDM4. While p21 was found (by us [12] and others, references within) to be a critical mediator of growth inhibition induced by MDM4 KD in BCs with wt p53, its protein level in response to MDM4 KD in TNBCs with mutant p53 were little affected (Figure 6A). P27 is a key cell cycle inhibitor that halts the progression of the cell cycle typically in G1 and was found to 
contribute to the cell cycle arrest imposed upon MDM4 KD in uveal melanoma with wt p53 [26]. In MDA-MB-468 cells, MDM4 KD induced p27 protein levels after three days (Figure 6A), but did not significantly affect its mRNA levels (Figure 6B). In accordance with p27 elevation, MDA-MB468 responded to MDM4 KD with growth inhibition at this three day time point (Figure 6C). Furthermore, p27 KD rescued the inhibition of cell proliferation that was induced by MDM4 KD (measured by trypan blue dye exclusion up to five days as shown by technical triplicates; Figure $6 \mathrm{C} ; \mathrm{p}=0.0191$ ). Data were confirmed in three independent experiments (averaged and normalised data for three experiments at day three; Figure 6D; $\mathrm{p}=0.0120$ ). Confirmation that MDM4 and $\mathrm{p} 27$ underwent $\mathrm{KD}$ at the five day experimental endpoint was demonstrated by immunoblotting (Figure 6E). Of note, MDM4 KD cells were less growth inhibited at day five than at day three, which corresponded with less p27 elevation. To further stratify the BC cell lines that respond to MDM4 KD by elevating p27, we studied additional lines (OCUB-M, SUM-159T, Hs 578T and CAMA-1). Only OCUB-M (Figure 6F) and MDA-MB-468 (Figure 6A) responded to MDM4 KD by elevating p27 levels and the absence of protein RB (pRB) from these cells distinguished them from the other four lines tested, which are reported to express pRB [27].

\section{Low p27, high MDM4 and p53 mutation are signatures of TNBCs.}

Low p27 has been frequently identified in TNBCs. To corroborate this in the context of MDM4, the basal-like TMA was stained for p27 (Figure 1A) and histochemistry scores were plotted alongside those for MDM4, p53 and MDM2 (Figure 1B). Correlation between relatively low p27 levels and high MDM4 was generally observed across the basal-like biopsies in the TMA $(p=0.0001)$. These findings offer another signature to discriminate a distinct set of TNBCs with potential therapeutic options. 


\section{DISCUSSION}

TP53 mutations in BC are selected in high-grade tumours that are aggressive and reduce survival. P53 mutations occur prior to metastatic disease and define the subset of tumour cells capable of dissemination (reviewed in [4]). This is consistent with the demonstrated gain of function of mutant p53 in the promotion of metastasis in mouse models $[28,29]$. This renders mutant p53 an attractive target for the treatment of aggressive BCs. Our study exposes strategic vulnerability in the dependency of BC with mutant p53 on the expression of MDM4, a key negative regulator of $\mathrm{p} 53$. We have recently described that MDM4 is highly expressed in a large proportion of luminal BCs, which are largely wt p53 [12]. This high expression of MDM4 can be rationalised in BCs expressing wt p53. Here, however, we show that MDM4 is also highly expressed in basal-like BCs, in which the majority of samples (64\%) express high p53 levels, suggestive of p53 mutations. Indeed, basal-like BCs (which encompasses most TNBCs; reviewed in [19]) are frequently mutated for $\mathrm{p} 53[2,9]$. These surprising findings triggered our search for an oncogenic role for MDM4 in BCs with mutant p53. Using a conditional KD approach, we found that MDM4 expression is essential for the proliferative capacity of multiple BC cell lines with mutant p53 (TNBCs: MDA-MB-468, MDA-MB-231, SUM-159PT, OCUM-B; Her2+: BT-474; and Luminal: CAMA-1). This also demonstrates that the effect of MDM4 KD is not limited to TNBCs, but also includes both luminal and Her2+ BCs with p53 mutation. Importantly, we have extended the finding to in vivo settings, in which tumour microenvironment impacts on growth. Using orthotopic xenograft models we show that MDM4 expression was critical both for the establishment of TNBC tumours and for tumour maintenance and growth. Therefore, while MDM4 KD inhibited tumour growth, eventual outgrowth of TNBC tumours was always associated with MDM4 re-expression, indicative of a selective defiance of MDM4 KD. Together, our studies demonstrate dependency of BC cells with 
mutant p53 on the expression of MDM4 for cell proliferation, tumour establishment and progression.

In BCs with wt p53 the oncogenic capacity of MDM4 to promote proliferative growth is achieved by curbing wt p53-mediated growth inhibition. This is mediated largely through p53-dependent transcriptional induction of p21, which promotes growth arrest and cellular senescence ([12] and references within). While mutant p53 does not induce p21 expression, MDM4 KD has been shown to promote the expression of three key players in the promotion of cell cycle arrest, pRb, p21 and p27, independently of p53 [26,30,31]. The p21 protein was not altered upon MDM4 KD in either MDA-MB-231 or MDA-MB-468 cells, so we focused on p27. Of interest for future investigation, of six TNBC cell lines studied, pRB null protein status $[27,32]$ was found to correlate with lines that elevated p27 in response to MDM4 KD (ie. MDA-MB-468 and OCUB-M). Furthermore, we identified a correlation between low p27 levels and high MDM4 expression in basal-like BC TMA biopsies with high p53 levels. MDM4 has been linked to p27 previously in a wt p53 context in uveal melanomas and p53-independent activity was postulated, although no mechanism was elucidated [26]. Of particular relevance, it has been proposed that low p27 protein levels constitute a signature that characterises aggressive BCs [32]. To what extent low p27 levels serve as a predictive marker for positive response to MDM4 targeting therapy will be important to evaluate in the future.

The therapeutic relevance of MDM4 depletion was demonstrated by a small molecular MDM4 inhibitor that has previously proven efficacious in uveal melanomas with wt p53 [26]. Consistent with inhibition of $B C$ cell proliferation in response to MDM4 KD, the drug also led to MDM4 depletion and corresponding growth inhibition of TNBC cells with 
mutant p53 (MDA-MB-468 and MDA-MB-231). The major focus on p53-related treatment has been directed to the development of MDM2 inhibitors [33], however these approaches had limited success due to severe 'on-target' effects which cause cytopaenia [34]. MDM4 may serve as a preferred target since its depletion does not markedly affect p53 levels ([35] and data shown here), MDM4 targeting is well tolerated in normal adult mouse tissues [36], and side effects were not observed in mice with MDM4 KD [37]. An important ramification from our findings is that MDM4 defines a rational drug target to treat aggressive, metastatic BCs with a poor prognosis, which are driven by p53 mutation.

\section{FIGURE LEGENDS}

Figure 1. MDM4 protein levels are high in Basal-like BCs with high levels of p53. (A) Representative images of IHC from the TMA of basal-like breast cancer biopsies ( $\mathrm{n}=87)$. Samples were stained for the indicated proteins (including MDM4 stained either with rabbit A300-287 or mouse monoclonal 8C6, with staining controls for p27 and MDM2). (B) Immunohistochemical scores of the p53 positive samples (56 of the 87cores) were plotted for staining of MDM4, p53, MDM2 and p27; ${ }^{* * *} \mathrm{p}=0.0001$, Student's t-test.

\section{Figure 2. MDM4 is detected in BC cell lines with mutant p53.}

(A) MDM4 protein (76kDa) was detected in BC lines across different subtypes using mouse monoclonal 8C6 for immunoblotting. Cell lines with mutant p53 were luminal, Her2+ or TNBC as indicated. MCF7 and MCF10A were used as controls for wt p53 cell lines. (B) MDM4-FL and MDM4-S mRNA were detected using semi-quantitative PCR. RPL37a was used as mRNA control.

Figure 3. MDM4 KD attenuates the growth of $\mathrm{BC}$ cells with mutant $\mathrm{p} 53$ in vitro. 
MDM4 KD was induced by Doxy treatment over 7 days (with supplementation on alternate days) and compared to shCtrls. MDM4 KD inhibited cell proliferation in BC cell lines with mutant p53 as measured by viable dye exclusion using flow cytometry. Levels of MDM4 were determined using 8C6 for immunoblotting of BC lines with mutant p53:

TNBC cell lines MDA-MB-468 (A, E). MDA-MB-231 (B, F) and SUM-159PT (C, G) and luminal cell line CAMA-1 (5 days; $\mathrm{D}, \mathrm{H}) .{ }^{* *} \mathrm{p}<0.01 ; * * * 0.001$, Student's t-test.

Figure 4. MDM4 KD inhibits the growth of TNBC xenografts with mutant p53 in vivo. (A, B) MDM4 KD induced by Doxy administration (indicated by blue arrows) inhibited the development of orthotopic xenografts and prolonged survival in a tumour establishment model of MDA-MB-468. (C - F) In a treatment model, MDM4 KD was induced when tumours reached $200 \mathrm{~mm}^{3}$ and tumour volume and survival measured for MDA-MB-468 and MDA-MB-231. (G) End point tumours from the treatment model for MDA-MB-468 with shCtrl and shMDM4 were stained for H\&E, Ki67, MDM4 (rabbit polyclonal A300287), p53, MDM2 and cleaved caspase-3 using serially sectioned FFPE samples. Detection of shRNA through the associated GFP marker is also shown from sectioned fresh frozen samples of the same tumours subjected to shMDM4 KD, compared with the shCtrls.

\section{Figure 5. The MDM4 targeting drug XI-011 inhibits cell growth of TNBC lines with} mutant p53. TNBC cell lines were exposed to XI-011 $(0.2 \mu \mathrm{M})$ over a period of 7 days and live cell numbers enumerated by flow cytometry at 3, 5 and 7 days (A and B; MDA-MB$468 \mathrm{p}=0.0018$ and MDA-MB-231 p=0.0007, respectively). Depletion of MDM4 in response to XI-011 was evident at day 6 as demonstrated using mouse monoclonal 8C6 for immunoblotting (C and D; MDA-MB-468 and MDA-MB-231 respectively). Statistics were assessed by Student's t-test and significance is indicated in the figures by: $*<0.05 ; * *<0.01$; 
$* * *<0.001$

Figure 6 KD of MDM4-mediated growth inhibition of TNBC cell lines with mutant p53 and lacking pRB expression is partially rescued by p27 depletion. (A) shRNAtransduced MDA-MB-468 and MDA-MB-231 cells were treated with Doxy for three days and the indicated proteins measured by immunoblotting. HSP60 was used as loading control. (B) mRNA levels of p27 were measured at the same time point. (C) KD of MDM4 and p27, alone or in combination, demonstrated by immunoblotting after 5 days of Doxy treatment in MDA-MB-468 and (D) the effect on cell proliferation at 3 and 5 days of Doxy treatment. (E) Average of 3 independent experiments of day 3 treatment. (F) Levels of MDM4, p27 and HSP60 in OCUB-M treated with Doxy for 5 days. MDM4 protein was detected in all immunoblots with 8C6. ** $\mathrm{p}<0.01$; Student's t-test.

\section{ACKNOWLEDGEMENTS}

This work was supported by NHMRC project grants (1049179) and NHMRC Fellowship to YH (9628426), by the CCV grant (1085154) and by an innovator grant from NBCF (IN-16-042).

\section{Author contributions:}

All the authors approved the submitted version of the papers, and either contributed to the drafts or critically revised it. In addition, JPM, DB, DR, EAT, AFAST, AP, J-M BP and TP performed and acquired data; MJH, CG, JCM, SF and AJ contributed to the design, analysis and interpretation of data. SH and YH conceptualized the project, wrote the manuscript and supervised.

This article is protected by copyright. All rights reserved. 


\section{ABBREVIATIONS}

Cycle Threshold $\left(\mathrm{C}^{\mathrm{T}}\right)$, Doxycycline (Doxy), Forward (fwd), Haematoxylin and Eosin staining (H\&E), intraperitoneal (IP), Knock down (KD), NOD SCID Gamma IL2R gamma chain (NSG), Reverse (rev), Tumour Microarray (TMA), Wild Type (wt)

\section{SUPPLEMENTARY MATERIAL ONLINE}

\section{Legends for Figures S1-S4}

Figure S1. MDM4 is detected by immunoblotting of a BC panel

Figure S2. Immunofluorescence staining indicates that mutant p53 and MDM4 localise to the same cellular regions in TNBCs

Figure S3. Multiple shMDM4 sequences reduce MDM4 protein levels and inhibit colony formation in the luminal BC line CAMA-1

Figure S4. MDM4 KD attenuates the growth of Her2+ and TNBC lines

Table S1. Cell line list

Table S2. Inducible lentiviral shRNA sequences

\section{REFERENCES}

1. Walerych D, Napoli M, Collavin L, et al. The rebel angel: mutant p53 as the driving oncogene in breast cancer. Carcinogenesis 2012; 33: 2007-2017.

2. Cancer Genome Atlas N. Comprehensive molecular portraits of human breast tumours. Nature 2012; 490: 61-70.

3. Brouckaert $\mathrm{O}$, Wildiers $\mathrm{H}$, Floris $\mathrm{G}$, et al. Update on triple-negative breast cancer: prognosis and management strategies. Int J Womens Health 2012; 4: 511-520.

4. Powell E, Piwnica-Worms D, Piwnica-Worms H. Contribution of p53 to metastasis. Cancer Discov 2014; 4: 405-414.

5. Zuckerman V, Wolyniec K, Sionov RV, et al. Tumour suppression by p53: the importance of apoptosis and cellular senescence. J Pathol 2009; 219: 3-15.

6. Selivanova G. Wild type p53 reactivation: from lab bench to clinic. FEBS Lett 2014; 588: 2628-2638.

7. Bieging KT, Mello SS, Attardi LD. Unravelling mechanisms of p53-mediated tumour suppression. Nat Rev Cancer 2014; 14: 359-370.

8. Forbes SA, Bindal N, Bamford S, et al. COSMIC: mining complete cancer genomes in the Catalogue of Somatic Mutations in Cancer. Nucleic Acids Res 2011; 39: D945950.

9. Shah SP, Roth A, Goya R, et al. The clonal and mutational evolution spectrum of primary triple-negative breast cancers. Nature 2012; 486: 395-399. 
10. Parise CA, Caggiano V. Breast Cancer Survival Defined by the ER/PR/HER2 Subtypes and a Surrogate Classification according to Tumor Grade and Immunohistochemical Biomarkers. J Cancer Epidemiol 2014; 2014: 469251.

11. Wade M, Li YC, Wahl GM. MDM2, MDMX and p53 in oncogenesis and cancer therapy. Nat Rev Cancer 2013; 13: 83-96.

12. Haupt S, Buckley D, Pang J-MB, et al. Targeting Mdmx to treat Breast Cancers with wild type p53. Cell Death Dis 2015; 6: e1821.

13. Lenos K, Grawenda AM, Lodder K, et al. Alternate splicing of the p53 inhibitor HDMX offers a superior prognostic biomarker than p53 mutation in human cancer. Cancer res 2012; 72: 4074-4084.

14. Wang H, Yan C. A small-molecule p53 activator induces apoptosis through inhibiting MDMX expression in breast cancer cells. Neoplasia 2011; 13: 611-619.

15. Herold MJ, van den Brandt J, Seibler J, et al. Inducible and reversible gene silencing by stable integration of an shRNA-encoding lentivirus in transgenic rats. Proc Natl Acad Sci (USA) 2008; 105: 18507-18512.

16. Louria-Hayon I, Grossman T, Sionov RV, et al. The promyelocytic leukemia protein protects p53 from Mdm2-mediated inhibition and degradation.J Biol Chem 2003; 278: 33134-33141.

17. Vichai V, Kirtikara K. Sulforhodamine B colorimetric assay for cytotoxicity screening. Nat Protoc 2006; 1: 1112-1116.

18. Skehan P, Storeng R, Scudiero D, et al. New colorimetric cytotoxicity assay for anticancer-drug screening. J Natl Cancer Inst 1990; 82: 1107-1112.

19. Badve S, Dabbs DJ, Schnitt SJ, et al. Basal-like and triple-negative breast cancers: a critical review with an emphasis on the implications for pathologists and oncologists. Mod Pathol Inc 2011; 24: 157-167.

20. Gembarska A, Luciani F, Fedele C, et al. MDM4 is a key therapeutic target in cutaneous melanoma. Nat Med 2012; 18: 1239-1247.

21. Touqan N, Diggle CP, Verghese ET, et al. An observational study on the expression levels of MDM2 and MDMX proteins, and associated effects on P53 in a series of human liposarcomas. BMC Clin Pathol 2013; 13: 32.

22. Haupt Y, Maya R, Kazaz A, et al. Mdm2 promotes the rapid degradation of $\mathrm{p} 53$. Nature 1997; 387: 296-299.

23. Kubbutat MH, Jones SN, Vousden KH. Regulation of p53 stability by Mdm2. Nature 1997; 387: 299-303.

24. Dewaele M, Tabaglio T, Willekens $\mathrm{K}$, et al. Antisense oligonucleotide-mediated MDM4 exon 6 skipping impairs tumor growth. J Clinical Invest 2016; 126: 68-84.

25. Lenos K, Jochemsen AG. Functions of MDMX in the modulation of the p53response. J Biomed Biotechnol 2011; 2011: 876173.

26. de Lange J, Teunisse AF, Vries MV, et al. High levels of Hdmx promote cell growth in a subset of uveal melanomas. Am J Cancer Res 2012; 2: 492-507.

27. Hollestelle A, Nagel JH, Smid M, et al. Distinct gene mutation profiles among luminal-type and basal-type breast cancer cell lines. Breast Cancer Res Treat 2010; 121: 53-64.

28. Lang GA, Iwakuma T, Suh YA, et al. Gain of function of a p53 hot spot mutation in a mouse model of Li-Fraumeni syndrome. Cell 2004; 119: 861-872.

29. Olive KP, Tuveson DA, Ruhe ZC, et al. Mutant p53 gain of function in two mouse models of Li-Fraumeni syndrome. Cell 2004; 119: 847-860.

30. Zhang H, Hu L, Qiu W, et al. MDMX exerts its oncogenic activity via suppression of retinoblastoma protein. Oncogene 2015. 
31. Jin Y, Zeng SX, Sun XX, et al. MDMX promotes proteasomal turnover of $\mathrm{p} 21$ at G1 and early S phases independently of, but in cooperation with, MDM2. Mol Cell Biol 2008; 28: 1218-1229.

32. Traub F, Mengel M, Luck HJ, et al. Prognostic impact of Skp2 and p27 in human breast cancer. Breast Cancer ResTreat 2006; 99: 185-191.

33. Khoo KH, Verma CS, Lane DP. Drugging the p53 pathway: understanding the route to clinical efficacy. Nat Rev Drug Discov 2014; 13: 217-236.

34. Burgess A, Chia KM, Haupt S, et al. Clinical overview of MDM2/X-targeted therapies. Front Oncol 2016; $6: 7$.

35. Marine JC, Jochemsen AG. MDMX (MDM4), a promising target for p53 reactivation therapy and beyond. Cold Spring Harb Perspect Med 2016; 6: a026237.

36. Garcia D, Warr MR, Martins CP, et al. Validation of MdmX as a therapeutic target for reactivating p53 in tumors. Genes Dev 2011; 25: 1746-1757.

37. Marine JC. Pharmacological rescue of p53 in cancer therapy: widening the sensitive tumor spectrum by targeting MDMX. Cancer Cell 2010; 18: 399-400.

This article is protected by copyright. All rights reserved. 


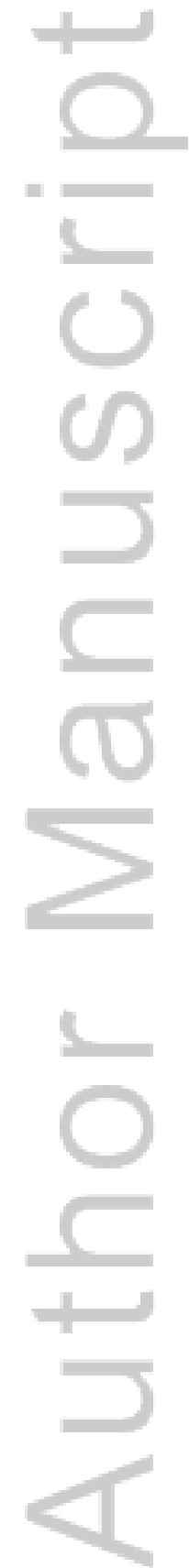

This article is protected by copyright. All rights reserved. 


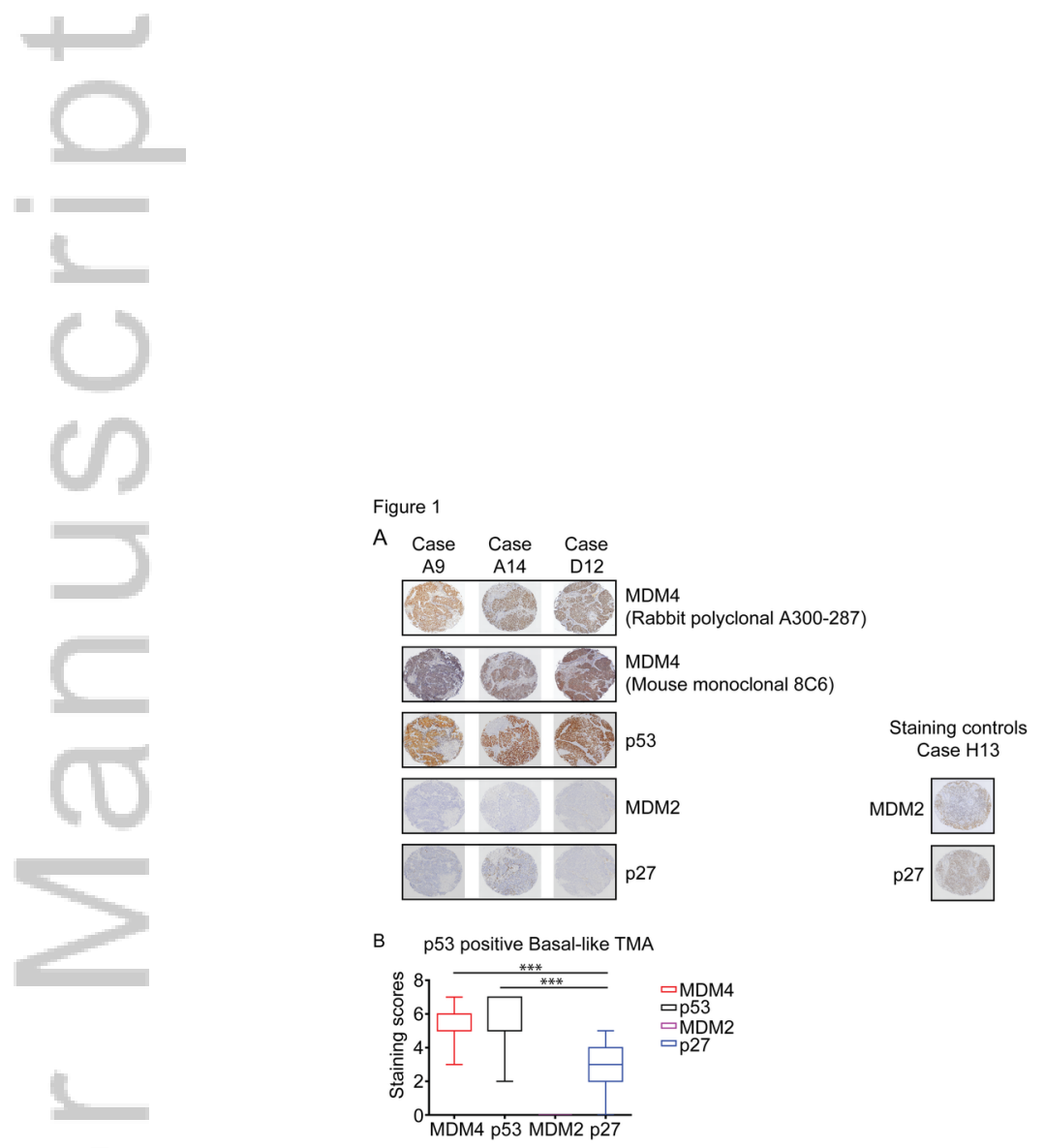

PATH_4877_Fig1.tif

This article is protected by copyright. All rights reserved. 


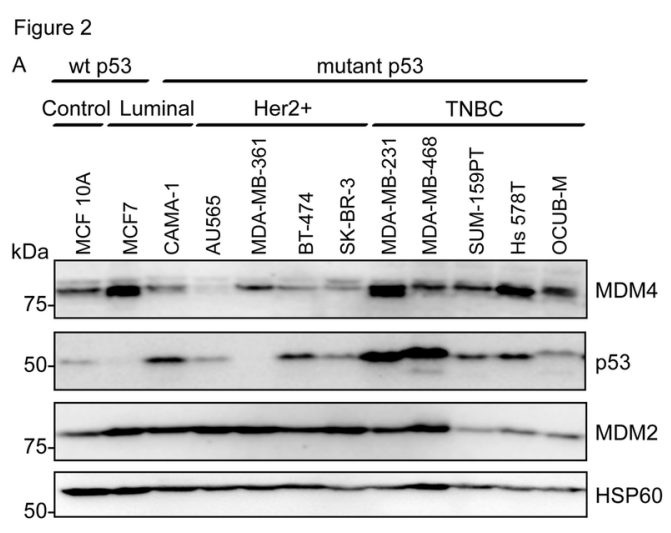

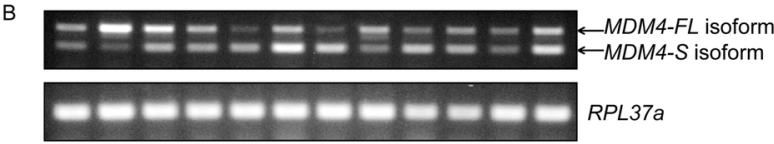

PATH_4877_Fig2.tif

This article is protected by copyright. All rights reserved. 


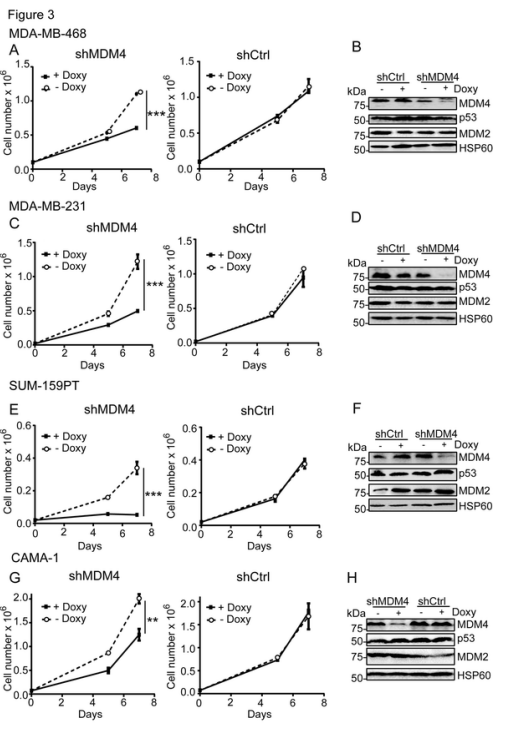

PATH_4877_Fig3.tif

This article is protected by copyright. All rights reserved. 

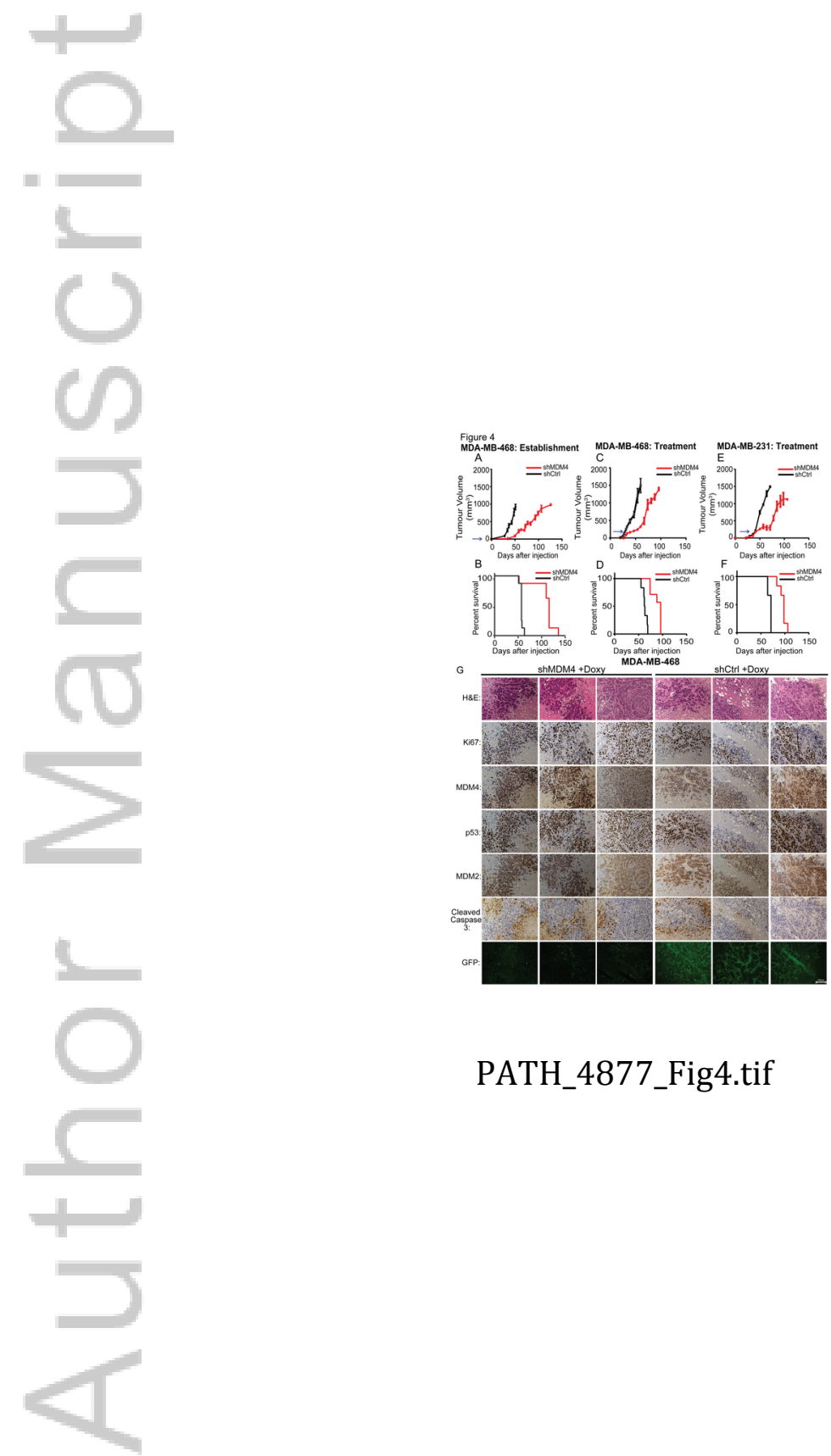

PATH_4877_Fig4.tif

This article is protected by copyright. All rights reserved. 


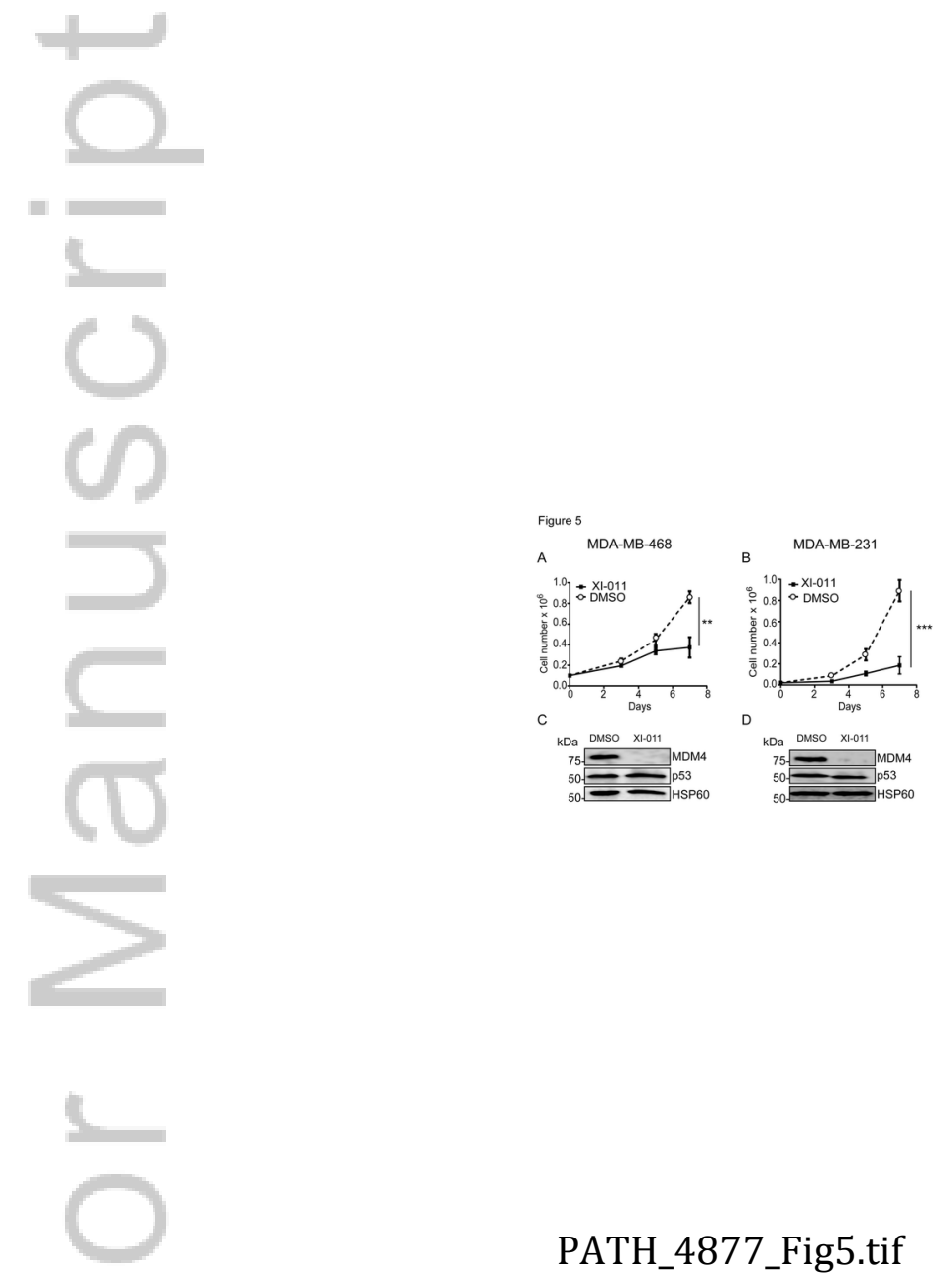

This article is protected by copyright. All rights reserved. 


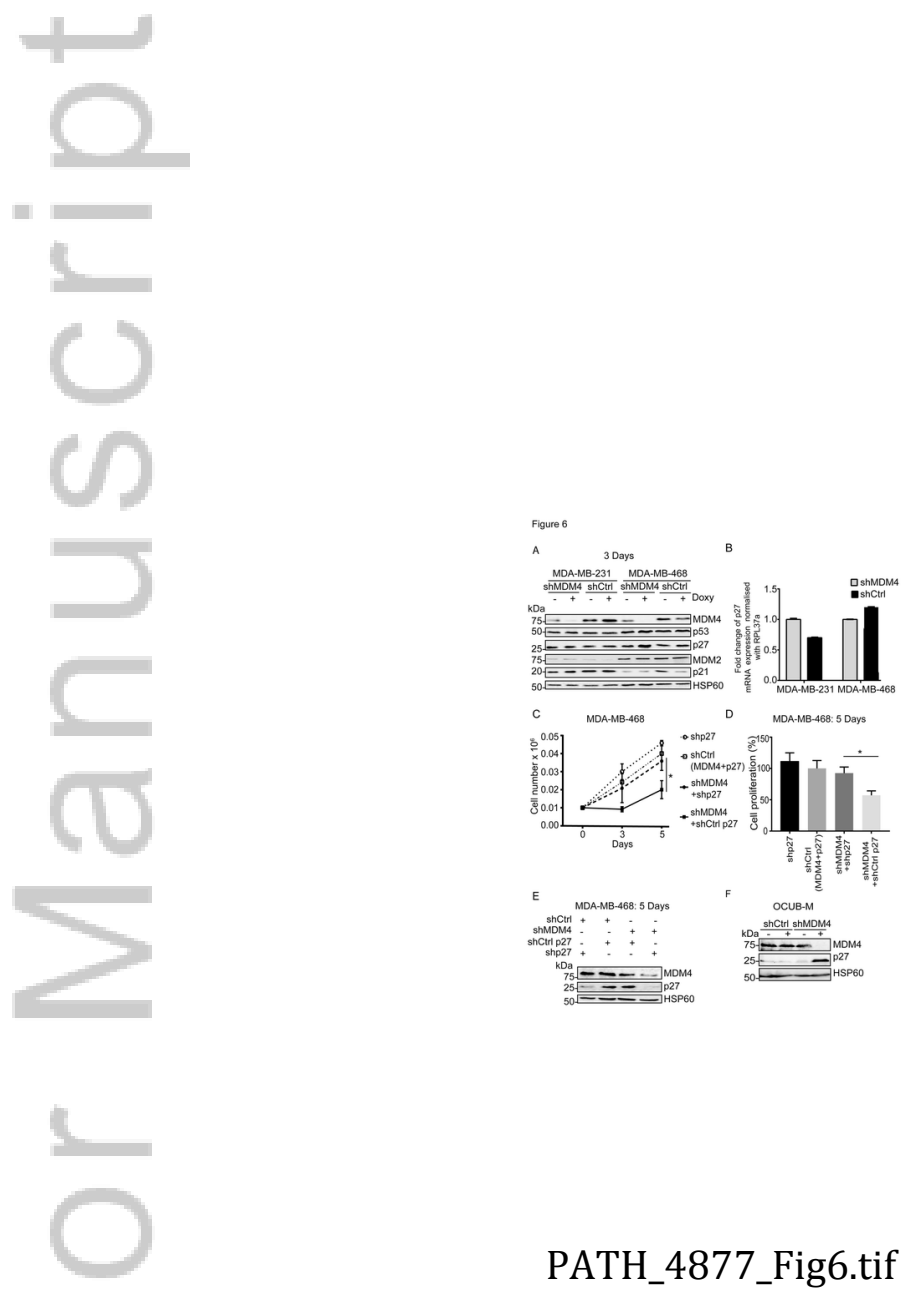

This article is protected by copyright. All rights reserved. 


\section{University Library}

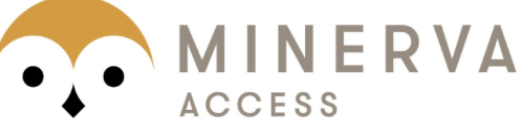

A gateway to Melbourne's research publications

Minerva Access is the Institutional Repository of The University of Melbourne

Author/s:

Miranda, PJ;Buckley, D;Raghu, D;Pang, J-MB;Takano, EA;Vijayakumaran, R;Teunisse, AFAS;Posner, A;Procter, T;Herold, MJ;Gamell, C;Marine, J-C;Fox, SB;Jochemsen, A;Haupt, S;Haupt, Y

Title:

MDM4 is a rational target for treating breast cancers with mutant p53

Date:

2017-04-01

Citation:

Miranda, P. J., Buckley, D., Raghu, D., Pang, J. -M. B., Takano, E. A., Vijayakumaran, R., Teunisse, A. F. A. S., Posner, A., Procter, T., Herold, M. J., Gamell, C., Marine, J. -C., Fox, S. B., Jochemsen, A., Haupt, S. \& Haupt, Y. (2017). MDM4 is a rational target for treating breast cancers with mutant p53. JOURNAL OF PATHOLOGY, 241 (5), pp.661-670. https:// doi.org/10.1002/path.4877.

Persistent Link:

http://hdl.handle.net/11343/292591 